\title{
EIGENVALUES AND EIGENVECTORS OF LATIN SQUARES IN MAX-PLUS ALGEBRA
}

\author{
Muhammad Syifa'ul Mufid ${ }^{1}$ And Subiono $^{2}$ \\ ${ }^{1,2}$ Institut Teknologi Sepuluh Nopember, Surabaya, Indonesia \\ syifaul.mufid11@mhs.matematika.its.ac.id, subiono2008@matematika.its.ac.id
}

\begin{abstract}
A Latin square of order $n$ is a square matrix with $n$ different numbers such that numbers in each column and each row are distinct. Max-plus Algebra is algebra that uses two operations, $\oplus$ and $\otimes$. In this paper, we solve the eigenproblem for Latin squares in Max-plus Algebra by considering the permutations determined by the numbers in the Latin squares.

Key words and Phrases: Latin squares, Max-plus Algebra, Eigenproblems, Permutation.
\end{abstract}

\begin{abstract}
Abstrak. Latin square order $n$ merupakan matriks persegi dengan $n$ angka berbeda sehingga angka-angka pada tiap baris dan kolom semuanya berbeda. Aljabar maxplus merupakan aljabar yang menggunakan dua operasi, $\oplus$ dan $\otimes$. Pada paper ini, diselesaikan permasalahan eigen dari Latin square pada aljabar max-plus dengan memperhatikan permutasi dari angka-angka pada Latin square tersebut.
\end{abstract}

Kata kunci: Latin square, Aljabar max-plus, Permasalahan eigen, Permutasi.

\section{INTRODUCTION}

In this paper we consider eigenproblems. From a square matrix $A$, eigenproblems are the problems of finding a scalar $\lambda$ and corresponding vector $v$ that satisfy $A v=\lambda v$ and we apply this problems into max-plus algebra. The problems can be solved by algorithm in [6]. The purpose of this paper is to solve eigenproblems in max-plus algebra for Latin squares by considering the permutations of symbol (or numbers) in Latin squares.

A reason for studying eigenproblems of Latin square in max-plus algebra is

2000 Mathematics Subject Classification: 05B15, 08A99, 15A18 Received: 14-08-2013, revised: 21-01-2014, accepted: 29-01-2014. 
that such problems have been studied for other matrices, for example Monge matrix [2], inverse Monge matrix [4] and circulant matrix [10, 11]. Eigenproblems are more simple to solve for that special matrices. For instance, eigenvalue of circulant matrices is equal to maximal number of that ones [10, 11].

The outline of this paper is as follows. In Section 2, we introduce Latin squares and permutations in the context of Latin squares. In Section 3, we introduce max-plus algebra and some theories about graph representation in max-plus algebra. Next in Section 4 we give theory of eigenproblems in max-plus algebra and some conditions to solve it. In Section 5 we give analyses to solve eigenproblems in max-plus algebra. In Section 6 we give an illustration of our problems. We give some remarks and conclusion in Section 7.

\section{Latin Square and Permutation}

A Latin square of order $n$ is a matrix of size $n \times n$ with $n$ different numbers such that in each row and each column filled by the permutation of those numbers [3], in other words the entries in each row and in each column are distinct [5]. Latin squares were firstly studied by Swiss mathematician, Leonhard Euler. The study of Latin square has long tradition in combinatorics [1], for example the enumeration of Latin squares. The method or formula to enumerate the number of Latin squares can be found in $[3,12,13]$. An example of Latin square of order 4 is shown in below Example 1

$$
L=\left[\begin{array}{llll}
2 & 3 & 1 & 4 \\
1 & 4 & 2 & 3 \\
4 & 1 & 3 & 2 \\
3 & 2 & 4 & 1
\end{array}\right]
$$

The notion of permutation is related to the act of rearranging objects or values. A permutation of $n$ objects is an arrangement of this objects into a particular order. For example there are six permutations of numbers $1,2,3$, that is $(1,2,3)$, $(1,3,2),(2,1,3),(2,3,1),(3,1,2)$ and $(3,2,1)$. For simplicity, we write a permutation without parentheses and commas. So we will write 123 rather than $(1,2,3)$. In this paper, we define $\underline{n}=\{1,2, \ldots, n\}$ as set of the $n$ first natural numbers.

In algebra, especially group theory, permutation is a bijective mapping on set $X$. A family of all permutations on $X$ is called the symmetric group $S_{X}$ [9], we write $S_{n}$ rather than $S_{X}$ for $X=\underline{n}$. From rearrangement $i_{1} i_{2} \ldots i_{n}$ of $\underline{n}$ we can define a function $\alpha: \underline{n} \rightarrow \underline{n}$ as $\alpha(1)=i_{1}, \alpha(2)=i_{2}, \ldots, \alpha(n)=i_{n}$. If $\alpha(i)=i$ for $i \in \underline{n}$, then $i$ is fixed by $\alpha$. For example, the rearrangement 321 determines the function $\alpha$ with $\alpha(1)=3, \alpha(2)=2, \alpha(3)=1$ and 2 fixed by $\alpha$.

We can write permutation in cycle form i.e. $\left(\begin{array}{llll}a_{1} & a_{2} & \ldots & a_{r}\end{array}\right)$ if $\alpha\left(a_{1}\right)=$ $a_{2}, \alpha\left(a_{2}\right)=a_{3}, \ldots, \alpha\left(a_{r-1}\right)=a_{r}, \alpha\left(a_{r}\right)=a_{1}$ and called by $r$-cycle (cycle of length $r)$. A complete factorization of a permutation $\alpha$ is a factorization of $\alpha$ into disjoint cycles that contains exactly one 1-cycle of $i$ for every $i$ fixed by $\alpha$ [9]. For example, the complete factorization of the 3-cycle $\alpha=\left(\begin{array}{lll}1 & 3 & 5\end{array}\right) \in S_{5}$ is $\alpha=\left(\begin{array}{lll}1 & 3 & 5\end{array}\right)(2)(4)$.

Suppose Latin square $L=\left(l_{i, j}\right)$ has order $n$. We can get $n$ permutations that represent of each number of $L$. Let $s \in \underline{n}$, we define permutation symbol of 
number $s$ by $\sigma_{s}$ such that $\sigma_{s}(i)$ equal to $j$ for which $l_{i, j}=s$ [12]. For example, from Latin square $L$ in Example 1, we get $\sigma_{1}, \sigma_{2}, \sigma_{2}, \sigma_{4} \in S_{4}$ as permutation symbol of number $1,2,3,4$ in $L$ respectively where $\sigma_{1}=\left(\begin{array}{lll}1 & 3 & 2\end{array}\right)(4), \sigma_{2}=\left(\begin{array}{lll}1 & (2 & 3\end{array}\right), \sigma_{3}=$ $\left(\begin{array}{lll}1 & 2 & 4\end{array}\right)(3), \sigma_{4}=\left(\begin{array}{lll}1 & 4 & 3\end{array}\right)(2)$.

\section{Max-Plus Algebra}

In max-plus algebra we define algebraic structure $\left(\mathbb{R}_{\varepsilon}, \otimes, \oplus\right)$, where $\mathbb{R}_{\varepsilon}$ is the set of all real numbers $\mathbb{R}$ extended by an infinite element $\varepsilon=-\infty$ and operation $\otimes, \oplus$ defined by

$$
x \oplus y=\max \{x, y\} \text { and } x \otimes y=x+y
$$

respectively. It is easy to show that both operation $\oplus$ and $\otimes$ are associative and commutative. Because $x \oplus \varepsilon=\varepsilon \oplus x=x$ and $x \otimes 0=0 \otimes x=x$ for all $x \in \mathbb{R}_{\varepsilon}$ then the null and unit element in max-plus algebra is $\varepsilon$ and 0 respectively.

For all $x \in \mathbb{R}_{\varepsilon}$ and non-negative integer $n$, we define

$$
x^{\otimes n}= \begin{cases}0, & \text { for } n=0 \\ \underbrace{x \otimes x \otimes x \otimes \ldots \otimes x}_{n}, & \text { for } n>0\end{cases}
$$

We can write $x^{\otimes n}$ in conventional algebra

$$
x^{\otimes n}=\underbrace{x \otimes x \otimes x \otimes \ldots \otimes x}_{n}=n \times x
$$

or generally for all $\beta \in \mathbb{R}$

$$
x^{\otimes \beta}=\beta \times x
$$

The set of all square matrices of order $n$ in max-plus algebra are defined by $\mathbb{R}_{\varepsilon}^{n \times n}$. Let $A \in \mathbb{R}_{\varepsilon}^{n \times n}$, the entry of $A$ at $i^{\text {th }}$ row and $j^{\text {th }}$ column is defined by $a_{i, j}$ and sometime we write $[A]_{i, j}$. For $A, B \in \mathbb{R}_{\varepsilon}^{n \times n}$, addition of matrix, $A \oplus B$, is defined by

$$
\begin{aligned}
{[A \oplus B]_{i, j} } & =a_{i, j} \oplus b_{i, j} \\
& =\max \left\{a_{i, j}, b_{i, j}\right\}
\end{aligned}
$$

and multiplication of matrix, $A \otimes B$, is defined by

$$
\begin{aligned}
{[A \otimes B]_{i, j} } & =\bigoplus_{k=1}^{n} a_{i, k} \otimes b_{k, j} \\
& =\max _{k \in \underline{n}}\left\{a_{i, k}+b_{k, j}\right\}
\end{aligned}
$$

For square matrix $A$, similar to scalar in max-plus algebra, we denote

$$
A^{\otimes k}=\underbrace{A \otimes A \otimes A \otimes \ldots \otimes A}_{k}
$$

as $k^{\text {th }}$ power of $A$.

From $L \in \mathbb{R}_{\varepsilon}^{n \times n}$, we can get directed graph (digraph) $\mathcal{G}(L)=\mathcal{G}(V, E)$, where $V$ is set of vertices and $E$ is set of edges. In $\mathcal{G}(L)$, there are $n$ vertices labelled by $1,2, \ldots, n$ respectively. There is an edge from vertex $i$ to vertex $j$ if $a_{j, i} \neq \varepsilon$ 
denoted by $(i, j)$. The weight of $(i, j)$-edge is denoted by $w(j, i)$ and equal to $a_{j, i}$, if $a_{j i}=\varepsilon$ then there is no $(i, j)$-edge. Graph representation of matrix $L$ in Example 1 is shown in Fig. 1.

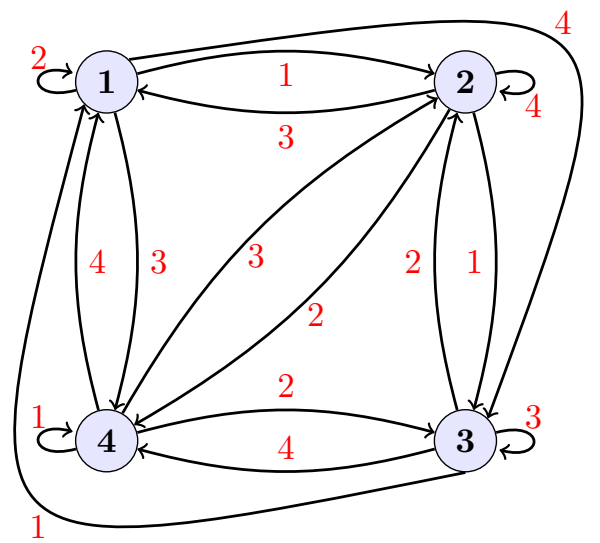

FIGURE 1. Graph representation of matrix $L$

A sequence of edges $\left(j_{1}, j_{2}\right),\left(j_{2}, j_{3}\right), \ldots,\left(j_{k-1}, j_{k}\right)$ is called path and if all vertices $j_{1}, j_{2}, \ldots, j_{k-1}$ are different then called elementary path. Circuit is an elementary closed path, i.e. $\left(j_{1}, j_{2}\right),\left(j_{2}, j_{3}\right), \ldots,\left(j_{k-1}, j_{1}\right)$. A circuit consists of a single edge, from a vertex to itself, is called a loop. Weight of a path $p=$ $\left(j_{1}, j_{2}\right),\left(j_{2}, j_{3}\right), \ldots,\left(j_{k-1}, j_{k}\right)$ is denoted by $|p|_{w}$ and equal to sum of all weight each edge i.e. $|p|_{w}=a_{j_{2} j_{1}}+a_{j_{3} j_{2}}+\ldots+a_{j_{k} j_{k-1}}$ and length of path is denoted by $|p|_{l}$ and equal to the number of edges in path $p$. The average weight of path $p$ defined by weight of $p$ divide by length of path $p$,

$$
\frac{|p|_{w}}{|p|_{l}}=\frac{a_{j_{2} j_{1}}+a_{j_{3} j_{2}}+\ldots+a_{j_{k} j_{k-1}}}{k-1}
$$

Any circuit with maximum average weight is called a critical circuit. A graph called strongly connected if there is a path for any vertex $i$ to any vertex $j$. If graph $\mathcal{G}(L)$ is strongly connected, then matrix $L$ is irreducible. We can infer that $[L]_{i, j}$ is equal to the weight of path with length 1 from $j$ to $i,\left[L^{\otimes 2}\right]_{i, j}$ is equal to the maximal weight of path with length 2 from $j$ to $i$ or generally for positive integer $k,\left[L^{\otimes k}\right]_{i, j}$ is equal to the maximal weight of path with length $k$ form $j$ to $i$.

There is relation between $\sigma_{i} \in S_{n}$ and a circuit in $\mathcal{G}(L)$. Every $r$-cycle in $\sigma_{i}$ represented circuit of length $r$ with each edge have weight $i$. Let graph representation in Fig. 1. We get $\sigma_{2}=(1)\left(\begin{array}{lll}2 & 3 & 4\end{array}\right)$ and there are two cycles of (1)(2 34 ), 1-cycle (1) and 3-cycle (2 34 ). As we can see in Fig. 1 there are two circuit with all edges have weight 2 , a loop in vertex 1 and a circuit with length 3 $(4,3),(3,2),(2,4)$. 
Let $A \in \mathbb{R}_{\varepsilon}^{n \times n}$, we define the matrix $A^{+}$as follow

$$
A^{+} \stackrel{\text { def }}{=} \bigoplus_{i=1}^{\infty} A^{\otimes i}=A \oplus A^{\otimes 2} \oplus \ldots \oplus A^{\otimes n} \oplus \ldots
$$

Because $\left[A^{\otimes k}\right]_{i, j}$ is equal to maximal weight of all paths with length $k$ from vertex $j$ to vertex $i$ then $\left[A^{+}\right]_{i, j}$ is equal to maximal weight of any path with any length from vertex $j$ to vertex $i$.

If $B \in \mathbb{R}^{n \times n}$ such that all circuits in $\mathcal{G}(B)$ have average weight less than or equal to 0 then $B^{+}$is equal to the summation (in max-plus) of $B^{\otimes k}$ for $k=$ $1,2, \ldots, n$, or in other words

$$
B^{+}=B \oplus B^{\otimes 2} \oplus \ldots \oplus B^{\otimes n}
$$

\section{EIGENPROBLEMS}

Eigenproblems are common problem in mathematics especially in linear algebra. In linear algebra, eigenproblems are the problems of finding $\lambda \in \mathbb{R}$ and vectors $v \in \mathbb{R}^{n}$ from matrix $A$ of size $n \times n$ that satisfy $A v=\lambda v$ and then $\lambda$ is called by eigenvalue while vector $v$ is called by eigenvector. In max-plus algebra, similar to linear algebra, eigenproblems are formulated as $A \otimes v=\lambda \otimes v$ for given matrix $A \in \mathbb{R}_{\varepsilon}^{n \times n}$, where $\lambda \in \mathbb{R}$ and $v \in \mathbb{R}^{n}$. The method to solve eigenproblems in max-plus algebra is quite different in linear algebra.

Methods to solve eigenproblems in max-plus algebra were handled by several authors for ordinary matrices $[6,7,8]$, as well as for special matrices such as circulant matrix [10, 11], Monge matrix [2] and inverse Monge matrix [4]. Special case for irreducible matrices, problem to get an eigenvalue related to problem to get critical circuits because the eigenvalue of $A$ is equal to the weight of critical circuits in $\mathcal{G}(A)$ [8]. If the eigenvalue exist for irreducible matrix $A$ then there is unique eigenvalue $[8]$.

In this paper we define $\lambda(A)$ as eigenvalue of matrix $A$ and $A_{\lambda}$ be a matrix such that $\left[A_{\lambda}\right]_{i, j}=[A]_{i, j}-\lambda(A)$ or in other word $A_{\lambda}=(-\lambda(A)) \otimes A$. It is clear that the maximum average weight of any circuit in $\mathcal{G}\left(A_{\lambda}^{+}\right)$is less than or equal 0 . Consequently, we can derived as follow

$$
A_{\lambda}^{+}=A_{\lambda} \oplus A_{\lambda}^{\otimes 2} \oplus \ldots \oplus A_{\lambda}^{\otimes n}
$$

and the $i^{\text {th }}$ column of $A_{\lambda}^{+}$is eigenvector of $A$ if $\left[A_{\lambda}^{+}\right]_{i, i}=0$ [8]. There is an algorithm to obtain eigenvalue and eigenvector that called Power Algorithm [6, 7].

\section{Discussion, Analyses and Results}

We will discuss Latin squares in max-plus algebra, so it is allowed to use infinite element $\varepsilon=-\infty$ as a symbol of a Latin square. Thus, we consider two cases of Latin squares.

- Case 1.

Latin square without infinite element that use $\underline{n}=\{1,2, \ldots, n\}$ as elements of Latin square. 
- Case 2.

Latin square with infinite element that use $\underline{n}_{\varepsilon}=\{\varepsilon, 1,2, \ldots, n-1\}$ as elements of Latin square

We denote $\mathcal{L}^{n}$ and $\mathcal{L}_{\varepsilon}^{n}$ be the set of all Latin squares of order $n$ without and with infinite element, respectively.

We begin the observation from graph representation of Latin square. Let $L_{1} \in \mathcal{L}^{n}$, because all numbers in $L_{1}$ are finite then $\left[L_{1}\right]_{i, j} \neq \varepsilon$ for all $i, j \in \underline{n}$ and it is clear that $\mathcal{G}\left(L_{1}\right)$ is strongly connected, consequently $L_{1}$ is irreducible. It can be concluded that all Latin squares without infinite element are irreducible matrix.

Let $L_{2} \in \mathcal{L}_{\varepsilon}^{n}$, because in each row and each column of $L_{2}$ there is exactly one $\varepsilon$ then $\left[L_{2}^{\otimes 2}\right]_{i, j}=\max _{k \in \underline{n}}\left\{a_{i, k}+a_{k, j}\right\}$ is finite. Consequently, there is a path length 2 from any vertex $i$ to any vertex $j$ and $L_{2}$ also irreducible. It can be concluded that all Latin squares with infinite element are irreducible matrix. Because both $L_{1}$ and $L_{2}$ are irreducible matrix then to find eigenvalue of $L_{1}$ and $L_{2}$ we need to find the critical circuit of graph representation of each matrix.

In next discussion we will solve eigenproblems of Latin squares in max-plus algebra and given the result about eigenvalue, eigenvector and the number of linearly independent eigenvectors also derive some theorems about them. See Section 6 for examples.

Theorem 5.1. Let $L_{1} \in \mathcal{L}^{n}$ and $L_{2} \in \mathcal{L}_{\varepsilon}^{n}$. The average weight of critical circuits of $\mathcal{G}\left(L_{1}\right)$ and $\mathcal{G}\left(L_{2}\right)$ is equal to $n$ and $n-1$ respectively.

Proof. We only need to consider permutation of the largest number in $L_{1}$ and $L_{2}$. It is clear that $\max \underline{n}=n$ and $\max \underline{n}_{\varepsilon}=n-1$. Let $\sigma_{n}$ be permutation symbol of number $n$ in $L$, from $\sigma_{n}$ we get circuit with the weight of all edges are $n$. Because all edges have weight $n$, then the average weight of circuit is $n$ and there is no circuit with average weight more than $n$. Thus, all circuits based on $\sigma_{n}$ are critical circuit in $\mathcal{G}\left(L_{1}\right)$ and the average weight of those critical circuit in $\mathcal{G}\left(L_{1}\right)$ is equal to $n$.

By the same argument, we get the average weight of critical circuits in $\mathcal{G}\left(L_{2}\right)$ is equal to $n-1$.

Theorem 5.2. Let $L_{1} \in \mathcal{L}^{n}$ and $L_{2} \in \mathcal{L}_{\varepsilon}^{n}$. Eigenvalue of $L_{1}$ and $L_{2}$ is equal to $n$ and $n-1$ respectively or generally eigenvalue of Latin square $L$ is equal to the maximal number in $L$.

Proof. The proof of this theorem is from direct result of Theorem 5.1

Let $L$ be Latin square of order $n$ that has eigenvalue $\lambda$. To get eigenvalue of Latin square in max-plus algebra we consider the matrix $L_{\lambda}^{+}$. We know that the $i^{\text {th }}$ column of $L_{\lambda}^{+}$is eigenvector of $L$ if $\left[L_{\lambda}^{+}\right]_{i, i}=0$. Number $i \in \underline{n}$ satisfies $\left[L_{\lambda}^{+}\right]_{i, i}=0$ if and only if in graph $\mathcal{G}(L)$ there is critical circuit from vertex $i$.

If $L$ is Latin square then $\lambda$ is equal to the maximal number in $L$ i.e. $\lambda(A)=$ 
$\max (A)$ and $\lambda$ appears exactly once in each row and column of $L$, consequently there is always critical circuit that every edge has weight $\lambda$ from any vertex $i$ for all $i \in \underline{n}$. Consequently, for Latin square $L$ all column of $L_{\lambda}^{+}$are eigenvector of $L$ with eigenvalue $\lambda$.

We say that two vectors $v_{1}, v_{2}$ are linearly independent (in max-plus algebra) if there is no $c \in \mathbb{R}$ such that $v_{1}=c \otimes v_{2}$. In max-plus algebra, it is possible that any matrix $L$ has two or more linearly independent eigenvectors.

We know that each critical circuit in $\mathcal{G}(L)$ represents eigenvector of $L$. If there are $m$ different critical circuits then there are $m$ linearly independent eigenvectors or we can say that the number of linearly independent eigenvectors is equal to the number of different critical circuit in $\mathcal{G}(L)$.

Theorem 5.3. Let $L$ a be Latin square with eigenvalue $\lambda$. The number of linearly independent eigenvectors of $L$ with respect to eigenvalue $\lambda$ is equal to the number of cycle in permutation symbol $\sigma_{\lambda}$.

Proof. Because $L$ is a Latin square with eigenvalue $\lambda$ then in graph $\mathcal{G}(L)$ there are critical circuits with average weight equal to $\lambda$ where each edge has weight $\lambda$. And because $\lambda$ appears exactly once in each row and column of $L$ then we can always make critical circuit based on permutation symbol of $\lambda$ i.e. $\sigma_{\lambda}$.

We know that every $r$-cycle in $\sigma_{\lambda}$ represented a critical circuit length $r$ where each edge have weight $\lambda$ then the number critical circuit is equal to the number of cycle in $\sigma_{\lambda}$ and this completes the proof.

\section{ExAmple}

We give two examples of Latin square, without and with infinite element $\varepsilon=-\infty$.

\section{Example I.}

$$
A=\left[\begin{array}{llll}
4 & 1 & 2 & 3 \\
1 & 2 & 3 & 4 \\
2 & 3 & 4 & 1 \\
3 & 4 & 1 & 2
\end{array}\right]
$$

By Theorem 5.2 eigenvalue of $A$ is maximal number in $A$ i.e. $\lambda(A)=\max (A)=4$. From $A$ we get permutation symbol $\sigma_{\lambda}=\sigma_{4}=(24)=(1)(24)(3) \in S_{4}$ and there are three cycles in $\sigma_{\lambda}$. Next we get

$$
\begin{aligned}
A_{\lambda} & =\left[\begin{array}{cccc}
0 & -3 & -2 & -1 \\
-3 & -2 & -1 & 0 \\
-2 & -1 & 0 & -3 \\
-1 & 0 & -3 & -2
\end{array}\right] A_{\lambda}^{\otimes 2}=\left[\begin{array}{cccc}
0 & -1 & -2 & -1 \\
-1 & 0 & -1 & -2 \\
-2 & -1 & 0 & -1 \\
-1 & -2 & -1 & 0
\end{array}\right] \\
A_{\lambda}^{\otimes 3} & =\left[\begin{array}{cccc}
0 & -1 & -2 & -1 \\
-1 & -2 & -1 & 0 \\
-2 & -1 & 0 & -1 \\
-1 & 0 & -1 & -2
\end{array}\right] A_{\lambda}^{\otimes 4}=\left[\begin{array}{cccc}
0 & -1 & -2 & -1 \\
-1 & 0 & -1 & -2 \\
-2 & -1 & 0 & -1 \\
-1 & -2 & -1 & 0
\end{array}\right]
\end{aligned}
$$


and

$$
\begin{aligned}
A_{\lambda}^{+} & =A_{\lambda} \oplus A_{\lambda}^{\otimes 2} \oplus A_{\lambda}^{\otimes 3} \oplus A_{\lambda}^{\otimes 4} \\
& =\left[\begin{array}{cccc}
0 & -1 & -2 & -1 \\
-1 & 0 & -1 & 0 \\
-2 & -1 & 0 & -1 \\
-1 & 0 & -1 & 0
\end{array}\right]
\end{aligned}
$$

By Theorem 5.3, the number of linearly independent eigenvectors is equal to the number of cycle in $\sigma_{\lambda}$ and from $A_{\lambda}^{+}$, we can get three different column vectors

$$
\left[\begin{array}{c}
0 \\
-1 \\
-2 \\
-1
\end{array}\right],\left[\begin{array}{c}
-1 \\
0 \\
-1 \\
0
\end{array}\right],\left[\begin{array}{c}
-2 \\
-1 \\
0 \\
-1
\end{array}\right]
$$

There are three linearly independent eigenvectors of $A$ with eigenvalue $\lambda=4$ and the number of cycle in $\sigma_{\lambda}$ is also 3 .

Example II.

$$
B=\left[\begin{array}{cccc}
2 & 3 & 1 & -\infty \\
3 & -\infty & 2 & 1 \\
1 & 2 & -\infty & 3 \\
-\infty & 1 & 3 & 2
\end{array}\right]
$$

By Theorem 5.2, the eigenvalue of $B$ is maximal number in $B$ i.e. $\lambda(B)=\max (B)=$ 3. From $B$ we get permutation symbol $\sigma_{\lambda}=\left(\begin{array}{lll}1 & 2\end{array}\right)\left(\begin{array}{ll}3 & 4\end{array}\right) \in S_{4}$ and there are two cycles in $\sigma_{\lambda}$. Next we get

$$
\begin{gathered}
B_{\lambda}=\left[\begin{array}{cccc}
-1 & 0 & -2 & -\infty \\
0 & -\infty & -1 & -2 \\
-2 & -1 & -\infty & 0 \\
-\infty & -2 & 0 & -1
\end{array}\right] B_{\lambda}^{\otimes 2}=\left[\begin{array}{cccc}
0 & -1 & -1 & -2 \\
-1 & 0 & -2 & -1 \\
-1 & -2 & 0 & -1 \\
-2 & -1 & -1 & 0
\end{array}\right] \\
B_{\lambda}^{\otimes 3}=\left[\begin{array}{cccc}
-1 & 0 & -2 & -1 \\
0 & -1 & -1 & -2 \\
-2 & -1 & -1 & 0 \\
-1 & -2 & 0 & -1
\end{array}\right] B_{\lambda}^{\otimes 4}=\left[\begin{array}{cccc}
0 & -1 & -1 & -2 \\
-1 & 0 & -2 & -1 \\
-1 & -2 & 0 & -1 \\
-2 & -1 & -1 & 0
\end{array}\right]
\end{gathered}
$$

and

$$
\begin{aligned}
B_{\lambda}^{+} & =B_{\lambda} \oplus B_{\lambda}^{\otimes 2} \oplus B_{\lambda}^{\otimes 3} \oplus B_{\lambda}^{\otimes 4} \\
& =\left[\begin{array}{cccc}
0 & 0 & -1 & -1 \\
0 & 0 & -1 & -1 \\
-1 & -1 & 0 & 0 \\
-1 & -1 & 0 & 0
\end{array}\right]
\end{aligned}
$$

By Theorem 5.3, the number of linearly independent eigenvectors is equal to the number of cycle in $\sigma_{\lambda}$ and from $B_{\lambda}^{+}$, we can get two different column vectors 


$$
\left[\begin{array}{c}
0 \\
0 \\
-1 \\
-1
\end{array}\right],\left[\begin{array}{c}
-1 \\
-1 \\
0 \\
0
\end{array}\right]
$$

There are two linearly independent eigenvectors of $B$ with eigenvalue $\lambda=3$ and the number of cycle in $\sigma_{\lambda}$ is also 2 .

\section{CONCLUSiON}

Eigenproblems for any Latin square $L$ can be solved by considering the permutation symbol of maximal number in $L$. Moreover, eigenvalue is equal to the maximal number in $L$ and the number of linearly independent eigenvectors is equal to the number of cycle in permutation symbol of those maximal number.

Acknowledgement. The authors would like to thank LPDP (Lembaga Pengelola Dana Pendidikan) for supporting this research by thesis scholarship.

\section{References}

[1] Denés, J., and Keedwell, A.D., Latin squares and their applications, Academic Press, New York, 1974.

[2] Gavalec, M., and Plavka, J., "Structure of The Eigenspace of a Monge Matrix in max-plus algebra", Discrete Applied Mathematics, 10 (2008), 596-606.

[3] Hulpke, A., Kaski, P., and Östergärd, P.R.J., "The Number of Latin Square of Order 11", Mathematics of Computation, 80:274 (2010), 1197-1219.

[4] Imaev, A.A., and Judd, R.P, "Computing an eigenvector of an inverse Monge matrix in max-plus algebra", Discrete Applied Mathematics, 158 (2010), 1701-1707.

[5] McKay, B.D., and Wanless, I.M., "On the Number of Latin Squares", Ann. Combinatorics, 9 (2005), 334-344.

[6] Subiono and van der Woude, J.W., "Power Algorithms for (max,+)- and Bipartite (min,max,+)-Systems", Discrete Event Dynamic Systems: Theroy and Application, 10 (2000), 369-389.

[7] Subiono, On Classes of Min-max-plus systems and Their Application, PhD Thesis, TU Delft, 2000.

[8] Subiono, Aljabar Max-plus dan Terapannya, Buku Ajar Mata Kuliah Pilihan Pasca Sarjana Matematika, Institut Teknologi Sepuluh Nopember, Surabaya, 2012.

[9] Rotmann, J.J., Advance Modern Algebra, Prentice Hall, New Jersey, 2003.

[10] Tomaskova, H., Eigenproblem for Circulant Matrices in Max-plus Algebra, University Hradec Kralove, Czech Republik, 2010.

[11] Ulfa, M., Analysis on Eigenvector of Circulant Matrix in Max-Plus Algebra (In Indonesian), Magister Thesis, Mathematics and Natural Science Faculty, Institut Teknologi Sepuluh Nopember, Surabaya, 2009.

[12] Stones, D.S., On the number of Latin rectangles, PhD Thesis, Monash University, 2010.

[13] Stones, D.S., "The Many Formulae for The Number of Latin Rectangles", Electronic Journal of Combinatorics, 17 (2010), \#A1, http://www.combinatorics.org/ojs/index.php/eljc/ article/view/v17ila1. 